\title{
Enhanced surface state protection and band gap in the topological insulator $\mathrm{PbBi}_{4} \mathrm{Te}_{4} \mathrm{~S}_{3}$
}

\author{
K. Sumida, ${ }^{1, *}$ T. Natsumeda, ${ }^{1}$ K. Miyamoto, ${ }^{2}$ I. V. Silkin, ${ }^{3}$ K. Kuroda, ${ }^{1,4}$ K. Shirai, ${ }^{1}$ S. Zhu,${ }^{1}$ K. Taguchi, ${ }^{1}$ M. Arita, ${ }^{2}$ \\ J. Fujii, ${ }^{5}$ A. Varykhalov, ${ }^{6}$ O. Rader, ${ }^{6}$ V. A. Golyashov, ${ }^{7,8,9}$ K. A. Kokh,,${ }^{7,8,10}$ O. E. Tereshchenko, ${ }^{7,8,9}$ \\ E. V. Chulkov, ${ }^{8,11,12}$ T. Okuda, ${ }^{2}$ and A. Kimura ${ }^{1, \dagger}$ \\ ${ }^{1}$ Graduate School of Science, Hiroshima University, 1-3-1 Kagamiyama, Higashi-Hiroshima 739-8526, Japan \\ ${ }^{2}$ Hiroshima Synchrotron Radiation Center, Hiroshima University, 2-313 Kagamiyama, Higashi-Hiroshima 739-0046, Japan \\ ${ }^{3}$ Tomsk State University, pr. Lenina 36, 634050 Tomsk, Russia \\ ${ }^{4}$ Institute for Solid State Physics, The University of Tokyo, 5-1-5, Kashiwa-no-ha, Chiba 277-8581, Japan \\ ${ }^{5}$ Istituto Officina dei Materiali (IOM)-CNR, Laboratorio TASC, in Area Science Park, S.S.14, Km 163.5, I-34149 Trieste, Italy \\ ${ }^{6}$ Helmholtz-Zentrum Berlin für Materialien und Energie, Elektronenspeicherring BESSY II, Albert-Einstein-Straße 15, 12489 Berlin, Germany \\ ${ }^{7}$ Novosibirsk State University, ul. Pirogova 2, 630090 Novosibirsk, Russia \\ ${ }^{8}$ Saint Petersburg State University, Saint Petersburg, 198504, Russia \\ ${ }^{9}$ Institute of Semiconductor Physics, Siberian Branch, Russian Academy of Sciences, pr. Akademika Lavrent'eva 13, \\ 630090 Novosibirsk, Russia \\ ${ }^{10}$ Institute of Geology and Mineralogy, Siberian Branch, Russian Academy of Sciences, Koptyuga pr. 3, 630090 Novosibirsk, Russia \\ ${ }^{11}$ Departamento de Fisica de Materiales UPV/EHU, CFM-MPC and Centro Mixto CSI-UPV/EHU, \\ 20080 San Sebastian/Donostia, Basque Country, Spain \\ ${ }^{12}$ Donostia International Physics Center, 20018 San Sebastian/Donostia, Basque Country, Spain
}

(Received 17 March 2018; published 1 October 2018)

\begin{abstract}
Topological insulators (TIs) with an inverted bulk band and a strong spin-orbit coupling exhibit gapless topological surface states (TSSs) protected by time-reversal symmetry. Helical spin textures driven by spinmomentum locking offer the opportunity to generate spin-polarized currents and therefore TIs are expected to be used for future spintronic applications. For practical applications TIs are urgently required that are operable at room temperature due to a wide bulk band gap as well as a distinct topological surface state that is robust to atmospheric exposure. Here we show two distinguishable TSSs originating from different terminations on $\mathrm{PbBi}_{4} \mathrm{Te}_{4} \mathrm{~S}_{3}$ by using spin- and angle-resolved photoemission spectroscopy. We find that one TSS is persistently observed, while the other becomes invisible upon intentional oxygen exposure. The result signifies the presence of a protected TSS buried under the topmost surface. Our finding paves the way for realizing a topological spintronics device under atmospheric conditions.
\end{abstract}

DOI: 10.1103/PhysRevMaterials.2.104201

\section{INTRODUCTION}

Two-dimensional electronic band structures at the surface and interfaces of topological insulators (TIs) are characterized by Fermi surface contours with helical locking of spins with momenta via spin-orbit coupling [1,2]. This has allowed the realization of novel basic spin-based phenomena, such as the inverse Edelstein effect that converts spin currents into a voltage $[3,4]$.

A practical application of TIs requires not only a high spin polarization but also additional physical protections of the topological surface state (TSS). In fact, a small amount of residual gas or water can easily induce band bending, which shifts the locus of a TSS [5-9]. Recently, the Pb-based ternary homologous series $\mathrm{PbBi}_{4} \mathrm{Te}_{7}$ and $\mathrm{PbBi}_{6} \mathrm{Te}_{10}$ have been theoretically proposed; they possess well-protected spin-polarized TSSs under a protecting layer [10,11]. These compounds are composed of a quintuple-layer (Te-Bi-Te-Bi-Te: $5 \mathrm{~L}$ ) and a

\footnotetext{
*sumida1126@hiroshima-u.ac.jp

†akiok@hiroshima-u.ac.jp
}

septuple-layer (Te-Bi-Te-Pb-Te-Bi-Te: 7L), which correspond to $\mathrm{Bi}_{2} \mathrm{Te}_{3}$ and $\mathrm{PbBi}_{2} \mathrm{Te}_{4}$ blocks, respectively. In this sense, two or three different terminations are possible that can form distinct TSSs.

Angle-resolved photoemission spectroscopy (ARPES) is very powerful at visualizing the band dispersions in solids. Previous ARPES experiments have identified two and three TSSs originating from different terminations for $\mathrm{PbBi}_{4} \mathrm{Te}_{7}$ and $\mathrm{PbBi}_{6} \mathrm{Te}_{10}$, respectively [11-13]. In particular, a study of $\mathrm{PbBi}_{4} \mathrm{Te}_{7}$ used depth-selective and spin-resolved ARPES to reveal that the TSS resides in the 7L block and is protected by the 5L block [12]. This tells us that the spin-polarized TSS is protected from external perturbations under the topmost layer. It can, however, rarely be used for practical devices working at room temperature because of the relatively narrow bulk band gap $(\sim 0.1 \mathrm{eV})$ in $\mathrm{PbBi}_{4} \mathrm{Te}_{7}$. One of the most efficient solutions for increasing the bulk band gap is to replace some heavy atoms with lighter isoelectronic analogs while maintaining the topological nature of the material. Furthermore, from an application point of view, experimental evidence is required that verifies whether the bulk energy gap is in fact enlarged via this process and whether the buried TSS survives when 


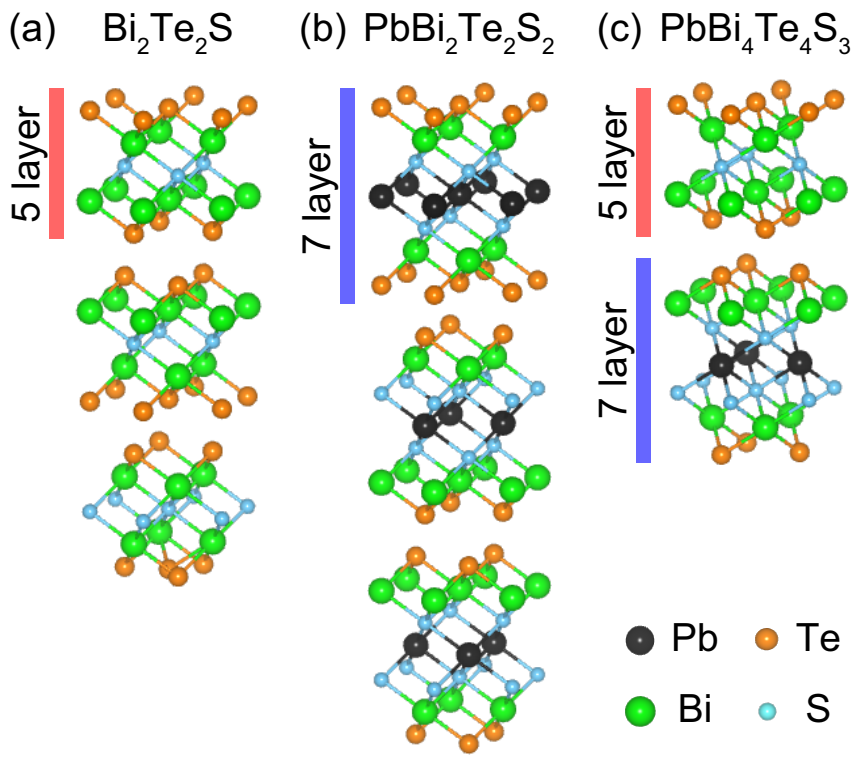

FIG. 1. Crystal structures of (a) $\mathrm{Bi}_{2} \mathrm{Te}_{2} \mathrm{~S}$, (b) $\mathrm{PbBi}_{2} \mathrm{Te}_{2} \mathrm{~S}_{2}$, and (c) $\mathrm{PbBi}_{4} \mathrm{Te}_{4} \mathrm{~S}_{3}$. Each block is separated by a van der Waals gap.

exposed to surface contamination. Recently, $\mathrm{PbBi}_{4} \mathrm{Te}_{4} \mathrm{~S}_{3}$ has been theoretically predicted to have a relatively wide bulk band gap $(\sim 0.3 \mathrm{eV})$ compared with $\mathrm{PbBi}_{4} \mathrm{Te}_{7}$, as well as a well-protected spin-polarized TSS [14]. $\mathrm{PbBi}_{4} \mathrm{Te}_{4} \mathrm{~S}_{3}$ possesses two different terminations in analogy with $\mathrm{PbBi}_{4} \mathrm{Te}_{7}$, where the 5L and 7L blocks are alternately stacked (Fig. 1).

In this work, we observed two distinct spin-polarized TSSs of $\mathrm{PbBi}_{4} \mathrm{Te}_{4} \mathrm{~S}_{3}$ using high-resolution ARPES with spin resolution. The presence of the protected TSS under the topmost layer was demonstrated upon intentional oxygen exposure. These experimental results can be reasonably explained by the calculated charge distributions of the TSSs with different terminations. Our finding paves the way for the realization of the spintronic devices that can operate under ambient pressure and temperature conditions.

\section{EXPERIMENT}

A single crystalline ingot of $\mathrm{PbBi}_{4} \mathrm{Te}_{4} \mathrm{~S}_{3}$ was grown from a nonstoichiometric composition using the vertical BridgmanStockbarger method. The atomic ratios of the constituent elements were determined via electron probe microanalysis. The samples were cleaved in situ in an ultrahigh vacuum with a base pressure of $1 \times 10^{-8} \mathrm{~Pa}$ at $<50 \mathrm{~K}$. High-resolution ARPES experiments with synchrotron radiation were conducted at the BL-9A beamline of the Hiroshima Synchrotron Radiation Center (HiSOR), Japan, the APE beamline of the ELETTRA Sincrotrone Trieste, Italy, and the UE112-PGM2a beamline of BESSY II, Germany. Spin-resolved ARPES (SARPES) experiments without and with an intentional oxygen exposure were performed at the ESPRESSO end station (BL-9B) of HiSOR [15,16]. Additionally, a microspot ultraviolet laser ARPES experiment was conducted at HiSOR. The energy and angular resolutions of ARPES (SARPES) were set to $<30 \mathrm{meV}$ and $<1^{\circ}\left(<60 \mathrm{meV}\right.$ and $\left.<3^{\circ}\right)$, respectively. The effective Sherman function was 0.28 for the SARPES
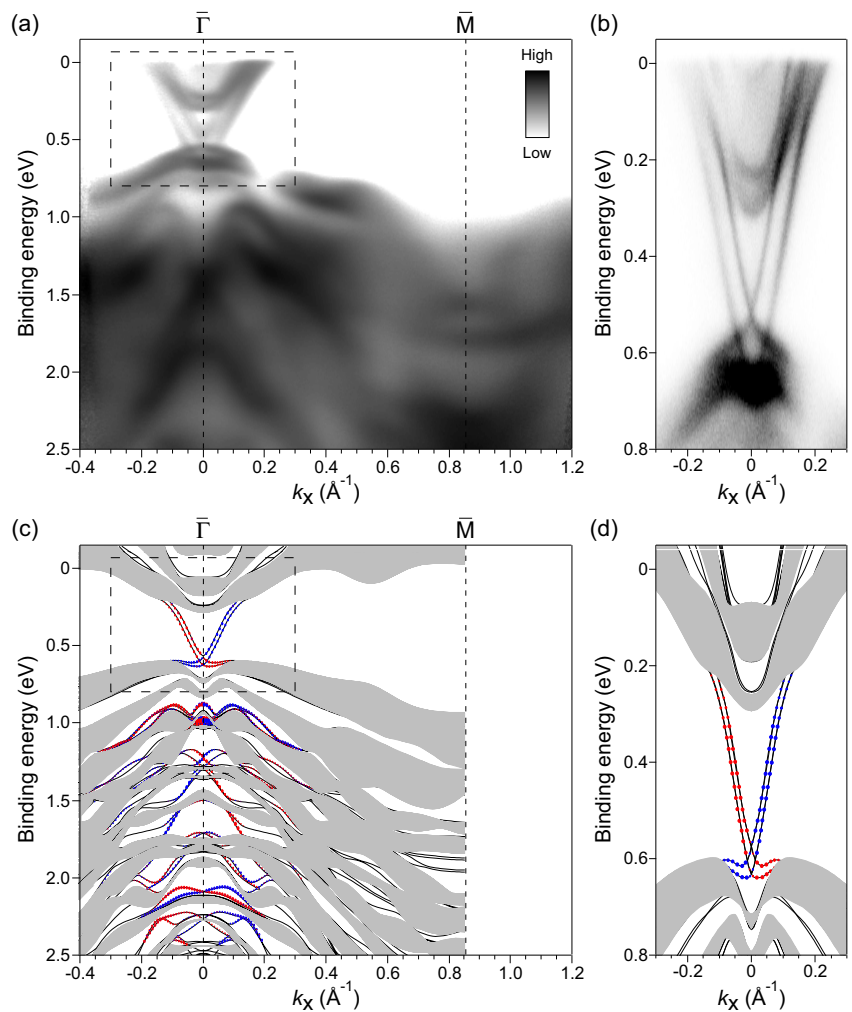

FIG. 2. Experimental band dispersions of $\mathrm{PbBi}_{4} \mathrm{Te}_{4} \mathrm{~S}_{3}$ in a wide (a) and narrow (b) energy and momentum range acquired at $h v=$ $18 \mathrm{eV}$ along the $\bar{\Gamma}-\bar{M}$ direction. The color intensity in (a) is presented in the logarithmic scale to show the photoemission signal more clearly. Panels (c) and (d) show the calculated surface (black solid lines) and bulk (gray area) band dispersions with both surface terminations. Red and blue circles represent the weight of in-plane spin polarization. Red (blue) color indicates positive (negative) spin polarization.

measurements. During the experiments, the temperature was kept below $50 \mathrm{~K}$. $A b$ initio calculations were performed within the framework of the density functional theory employing a pseudopotential method implemented in the VASP code $[17,18]$. For description of the exchange-correlation effects, the generalized gradient approximation was used in the PBE parametrization [19].

\section{RESULTS AND DISCUSSION}

Figure 2(a) shows the wide energy- and momentum-range ARPES image along the $\bar{\Gamma}-\bar{M}$ line recorded at $h v=18 \mathrm{eV}$ with $p$ polarization. In the vicinity of the $\bar{\Gamma}$ point, we confirmed Dirac-cone-like band dispersion near the Fermi level $\left(E_{\mathrm{F}}\right)$, as denoted with a box in Fig. 2(a). At the higher binding energy $\left(E_{\mathrm{B}}=0.9-1.5 \mathrm{eV}\right)$, we also found an M-shaped band structure with an energy maximum of $E_{\mathrm{B}}=0.9 \mathrm{eV}$ and a wave number of $\pm 0.13 \AA^{-1}$. This likely indicates a Rashbatype surface state, which is induced by a strong spin-orbit coupling [20,21]. To take a closer look at the Dirac-cone-like band dispersion, a high-resolution ARPES image was taken, as shown in Fig. 2(b). Here we can observe two distinct Dirac cones. The Dirac point of the inner cone is located at $0.52 \mathrm{eV}$ 

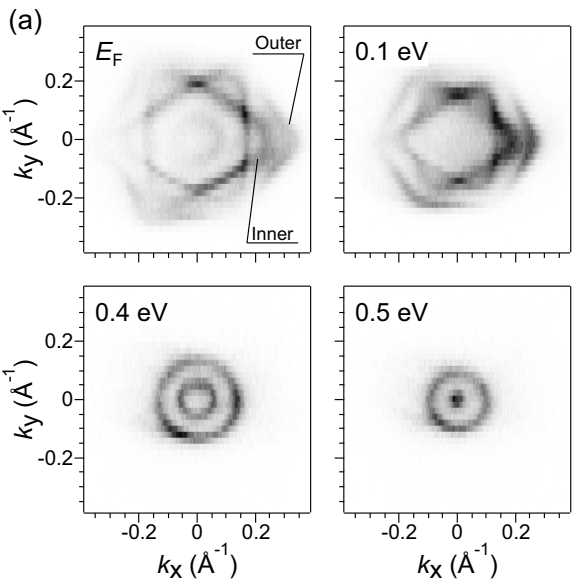
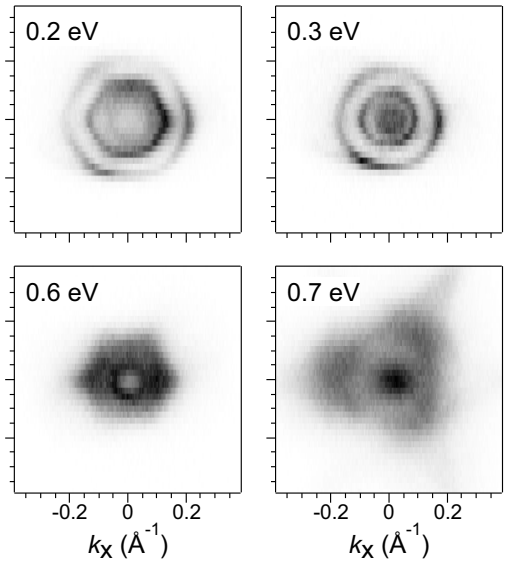

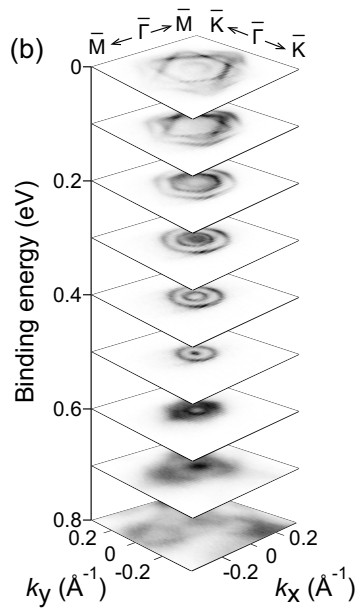

FIG. 3. (a) Constant energy contours taken at $E_{\mathrm{B}}=0,0.1,0.2,0.3,0.4,0.5,0.6$, and $0.7 \mathrm{eV}$. The loci of the Dirac points for the inner and outer Dirac cones were estimated to be $E_{\mathrm{B}} \sim 0.5$ and $0.7 \mathrm{eV}$, respectively. (b) Corresponding three-dimensional map recorded at $h v=18 \mathrm{eV}$.

and we can clearly see both the upper and lower part of the Dirac cones. Besides, the locus of the outer cone is slightly lower than the inner cone. In Figs. 2(c) and 2(d), the calculated band dispersions at both surface terminations are shown in the same energy and momentum range used in Figs. 2(a) and 2(b). The top of the bulk valence and the bottom of the bulk conduction bands are located around \pm 0.1 and $0 \AA^{-1}$, respectively. The bulk band gap was experimentally estimated to be approximately $0.2 \mathrm{eV}$ (see Supplemental Material [22]). Here, one can clearly see that the two TSS branches connect the parabolic conduction band and the M-shaped bulk valence band. They are almost linear above their Dirac points in the bulk energy gap region, while their slope becomes a little less steep when they get into the bulk conduction band energy region. These features are quite consistent with those in the experimental ARPES image [Fig. 2(b)]. There is a little discrepancy, however, in the lower part of the inner TSS. Namely, the dispersion is almost linear with a less steep dispersion than that above the Dirac point in the experiment, while the calculated TSS shows an upturn toward the higher $\left|k_{\mathrm{x}}\right|$. This is probably related to the feature that the predicted Dirac point of the inner TSS is located closer to the valence band maximum than that of the experimental one. Nevertheless, it can be safely said that the locus of the Dirac point of the inner TSS is still higher than that of the bulk valence maximum, which is consistent with the experimental result. In contrast, the outer TSS has its Dirac point below the valence band maximum that leads to an upward dispersion as seen both experimentally and theoretically. Then we can conclude that the Dirac point $\left(0.52 \mathrm{eV}\right.$ below $\left.E_{\mathrm{F}}\right)$ of the inner TSS is located at $0.015 \mathrm{eV}$ above the valence band maximum, while that of the outer TSS is buried inside the valence band maximum.

Although the estimated bulk band gap is smaller than the theoretically predicted value [14], it is more than twice as large as the gap size of $\mathrm{PbBi}_{4} \mathrm{Te}_{7}$ [12]. The key to increase the bulk band gap is to replace the heavy element (Te) with light element (S), which shrinks the lattice constant from $(a, c)=(4.418,23.948) \AA$ to $(4.241,23.121) \AA[11,23]$. Note that the loci of both Dirac points are much deeper than the $E_{\mathrm{F}}$; these energy shifts are attributed to an unintentional doping by impurities or defects as observed for $\mathrm{Bi}_{2} \mathrm{Se}_{3}$ and $\mathrm{Bi}_{2} \mathrm{Te}_{3}[24,25]$ but the bulk carrier would be controlled by the replacement of $\mathrm{Sb}$ for $\mathrm{Bi}[26,27]$. An appropriate bulk carrier tuning to locate the $E_{\mathrm{F}}$ in the bulk band gap would enable one to avoid thermal carrier excitation at room temperature.

Figure 3 shows the constant energy contours taken at several binding energies. At $E_{\mathrm{F}}$, we can see a complex Fermi surface deformed into a hexagon. In going from the Dirac points to $E_{\mathrm{F}}$, the distribution pattern gradually evolves from circular to hexagonal. This evolution is caused by the warping effect, which becomes pronounced at energies away from the Dirac point [28]. This deformation starts from around $E_{\mathrm{B}} \sim$ $0.3 \mathrm{eV}$, below which the Dirac cone energetically overlaps with the bulk conduction band; most importantly, almost circular-shaped contours were observed for both Dirac cones in the bulk energy gap region [see contours at $E_{\mathrm{B}}=0.4$, $0.5 \mathrm{eV}$ in Fig. 3(a)].

To confirm whether the observed Dirac-cone-like band dispersions are caused by two different terminations, we performed high spatial resolution ARPES by using a laser light source. Figure 4(a) shows photographs taken from the cleaved sample. The markers A and B in the lower panel of Fig. 4(a) indicate the measurement points. Figures 4(b) and 4(c) show the ARPES images and their second derivatives taken from the surfaces $\mathrm{A}$ and $\mathrm{B}$, respectively, depicted in the lower panel of Fig. 4(a). Owing to the small spot size $(<10 \mu \mathrm{m})$, we separately observed the two different band dispersions by slightly changing the measurement position. This strongly indicates that the two Dirac cones stem from different surface domains with independent band dispersions owing to different terminations. Then we estimated the energy positions of the Dirac points for the surfaces A and B, which correspond to the outer and inner Dirac cones, to be $E_{\mathrm{B}}=$ 0.64 and $0.52 \mathrm{eV}$, respectively. We found that the Dirac point is located below the top of the bulk valence band at surface A. Conversely, the Dirac point of surface B appeared to be well isolated from the bulk band and is similar in shape to the band dispersion of $\mathrm{PbBi}_{2} \mathrm{Te}_{4}$ [27,29]. These features are in good agreement with the results of the first-principles calculations shown in Figs. 2(c) and 2(d). This indicates that 

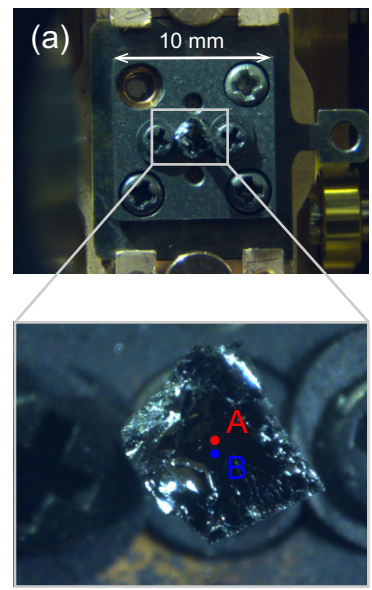

(b)

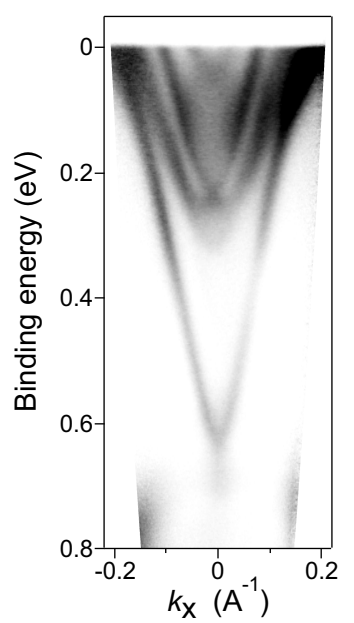

Surface A

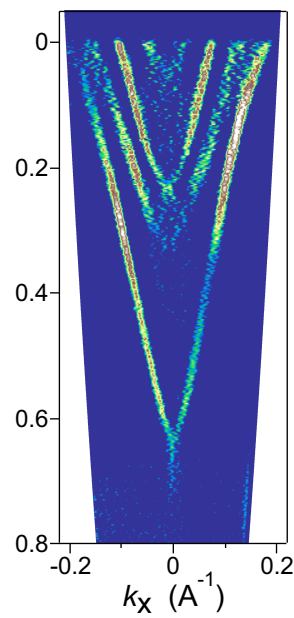

(c)

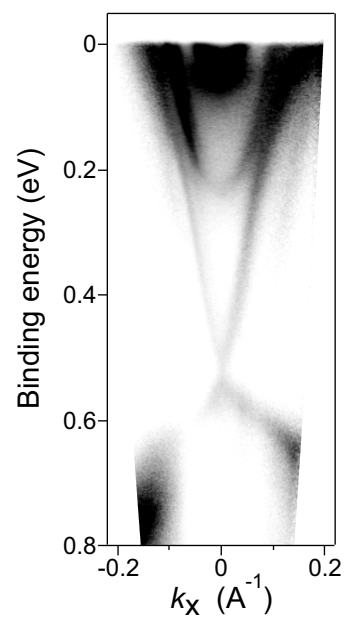

Surface B

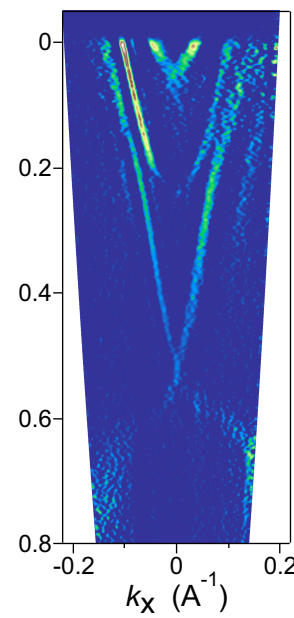

FIG. 4. (a) Photographs of the cleaved sample. The measurement points are depicted as surface A and B in the lower panel. Panels (b) and (c) show ARPES images and their second derivatives along the $\bar{\Gamma}-\bar{M}$ direction recorded at $h v=6.33$ with $s$ polarization from the surfaces A and $\mathrm{B}$, respectively. The second derivatives were taken along the momentum directions.

surfaces $\mathrm{A}$ and $\mathrm{B}$ correspond to those terminated with the $5 \mathrm{~L}\left(\mathrm{Bi}_{2} \mathrm{Te}_{2} \mathrm{~S}\right)$ and the $7 \mathrm{~L}\left(\mathrm{PbBi}_{2} \mathrm{Te}_{2} \mathrm{~S}_{2}\right)$ blocks, respectively. Next, we estimated the Dirac velocities for the outer and inner Dirac cones to be $\sim 3.7 \times 10^{5}$ and $\sim 3.9 \times 10^{5} \mathrm{~m} / \mathrm{s}$, (a)
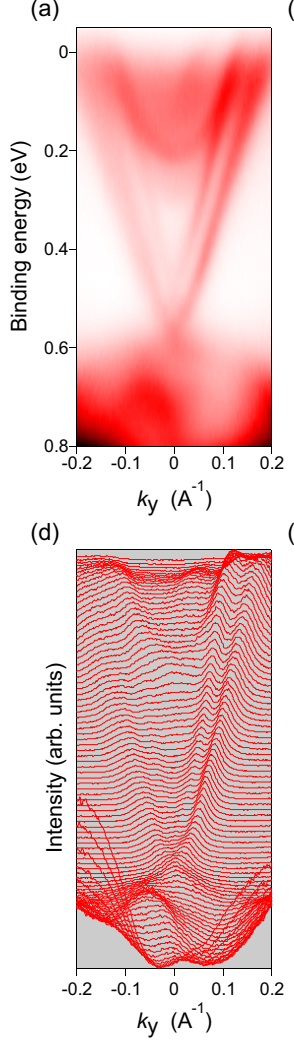

(b)

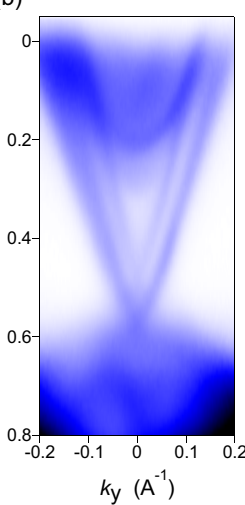

(c)

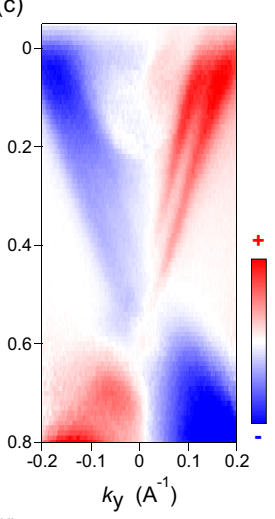

(e)

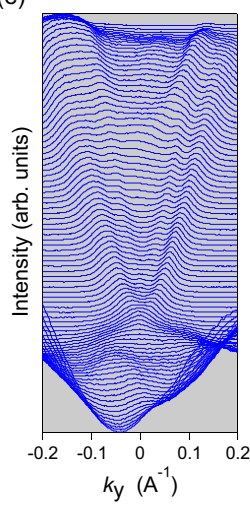

(f)

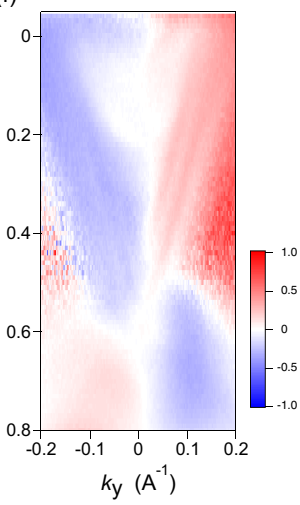

FIG. 5. In panels (a) and (b) ARPES images along the $\bar{\Gamma}-\bar{K}$ direction recorded at $h v=15 \mathrm{eV}$ with right $(C+)$ and left $(C-)$ circularly polarized light, respectively, are shown. (c) Difference image defined as $I_{C+}-I_{C-}$. Panels (d) and (e) show the MDCs of the ARPES images in panels (a) and (b), respectively. (f) Normalized difference image defined as $\left(I_{C+}-I_{C-}\right) /\left(I_{C+}+I_{C-}\right)$. respectively. These Dirac velocities are relatively faster than those of the other $\mathrm{Pb}$ contained TIs $[13,29]$. In addition, we found Rashba-like states at surface A; these states overlap energetically with the bulk conduction band [Fig. 4(b)]. This feature is sometimes observed in aged TIs owing to residual gas adsorption [7,9].

To gain insight into the spin and orbital entangled features in the surface states and to confirm their topological origins, we first investigated the circular dichroism in the angular distribution (CDAD) of the photoelectrons (see Supplemental Material [22]). The CDAD is proposed to allow the spin and/or orbital angular momenta for the initial state in the strong spin-orbit coupled system to be obtained [30-33]. Figures 5(a) and 5(b) show the ARPES images, while Figs. 5(d) and 5(e) show their corresponding momentum distribution curves (MDCs) recorded with right $(C+)$ and left $(C-)$ handed circularly polarized light, respectively. To emphasize (a) $\mathrm{hv}=26 \mathrm{eV}$

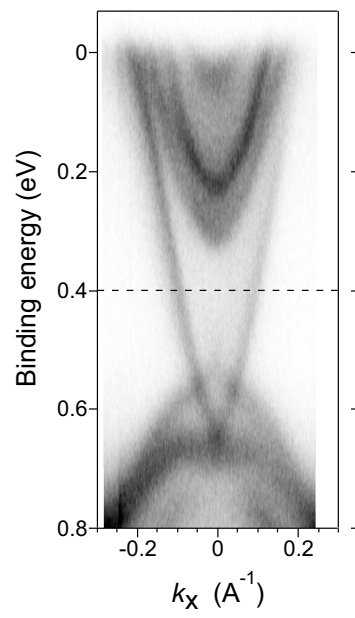

(b) $\mathrm{hv}=28 \mathrm{eV}$

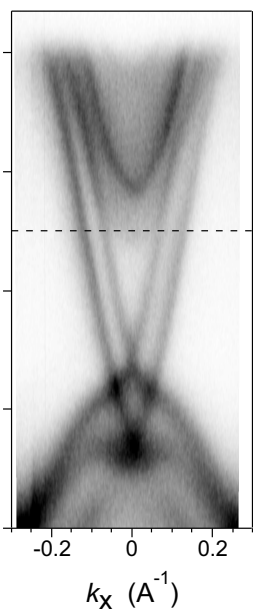

(c) $\mathrm{hv}=30 \mathrm{eV}$

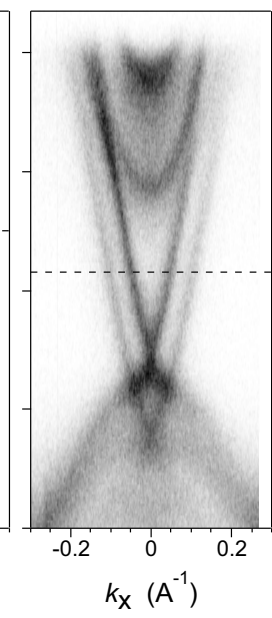

FIG. 6. Photon-energy dependence. Band dispersions along the $\bar{\Gamma}-\bar{M}$ direction recorded at $h v=26$ (a), 28 (b), and $30 \mathrm{eV}$ (c) with $p$ polarization. 
(a)

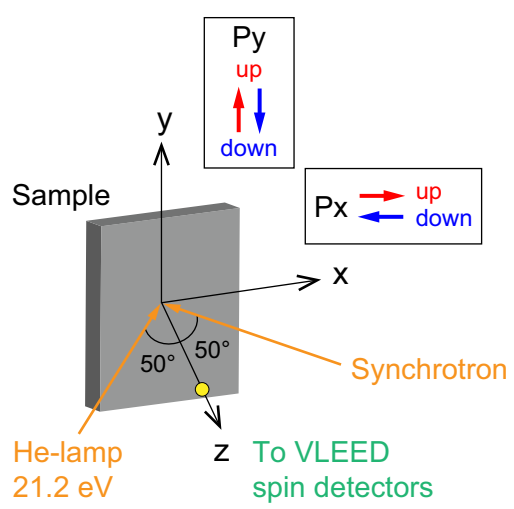

(b)

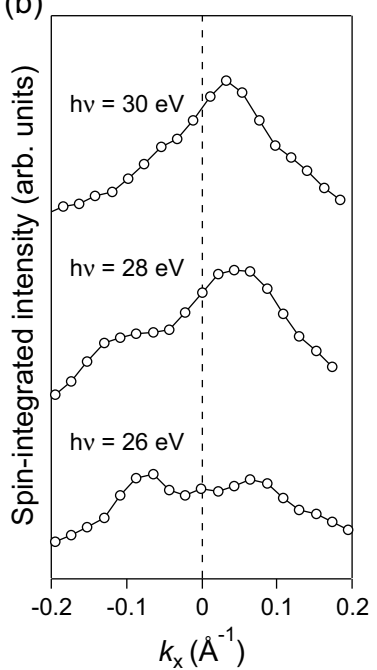

(c)

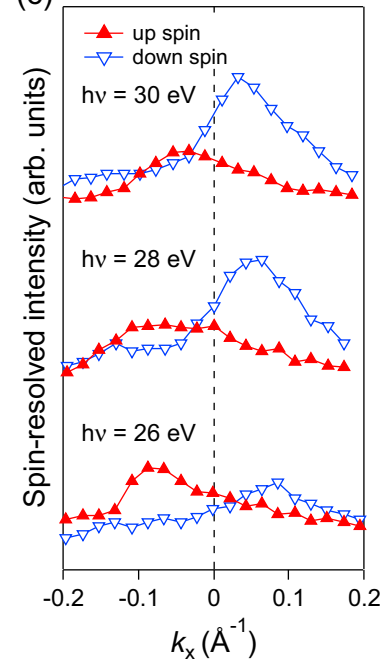

(d)

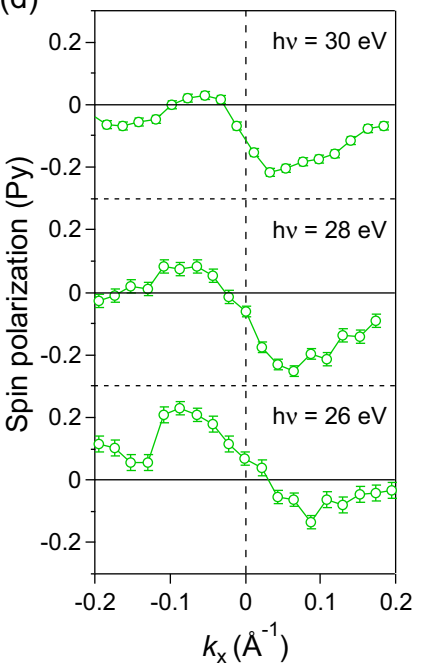

FIG. 7. (a) Experimental geometry for SARPES. Spin quantized axes are also shown in (a). Spin-integrated (b) and spin-resolved MDCs (c) and their spin polarizations (d) along the $\bar{\Gamma}-\bar{M}$ direction taken at $h v=26,28$, and $30 \mathrm{eV}$ for $E_{\mathrm{B}}$ of $0.40,0.30$, and $0.37 \mathrm{eV}$.

the difference between the two images, we have adopted two methods. Namely, the difference $I_{C+}-I_{C-}$ and the normalized difference $\left(I_{C+}-I_{C_{-}}\right) /\left(I_{C+}+I_{C_{-}}\right)$method; the results are shown in Figs. 5(c) and 5(f), respectively. In both cases, clear CDAD can be seen. The sign of the CDAD is reversed with respect to the $\bar{\Gamma}$ point. Furthermore, the signs of the CDAD at the inner and outer Dirac cones are the same, which may imply that both Dirac cones have the same spin orien- tations or orbital characteristics. Note that the clear CDAD was observed not only in the Dirac cones but also in the bulk valence and conduction bands. This is probably attributed to a strong spin-orbit coupling or a geometrical effect [34,35].

To clarify the difference between the two distinct Dirac cones, we performed photon-energy dependent ARPES measurements. Figure 6 shows the band dispersions recorded at photon energies of $h v=26,28$, and $30 \mathrm{eV}$. At $h v=28 \mathrm{eV}$ (a)

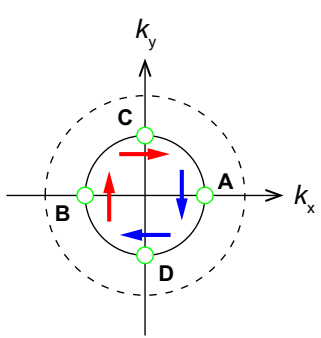

(b)

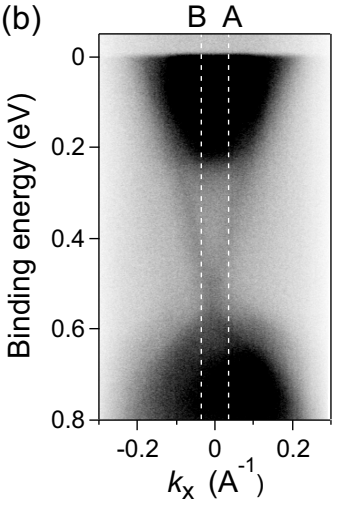

(c)

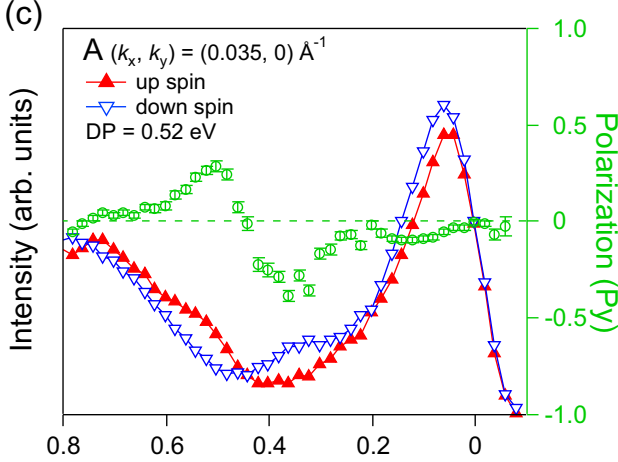

(d)

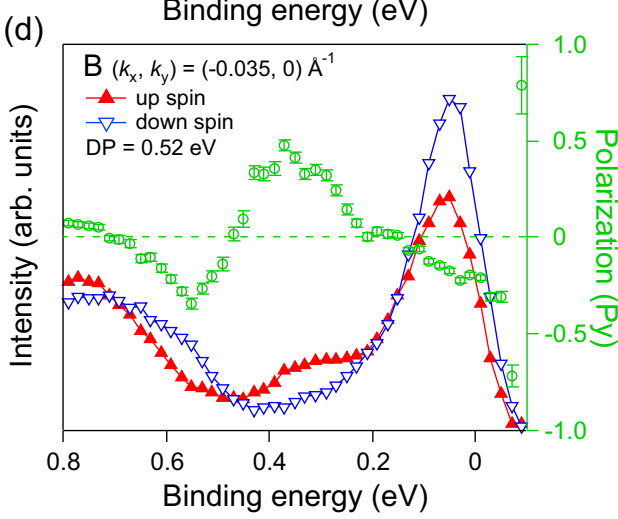

(e)

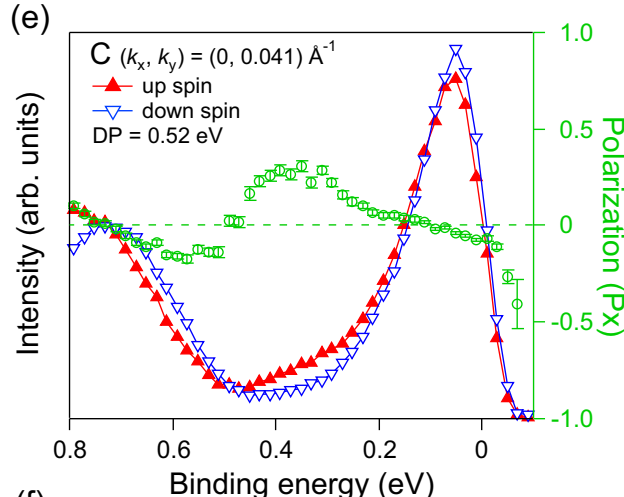

(f)

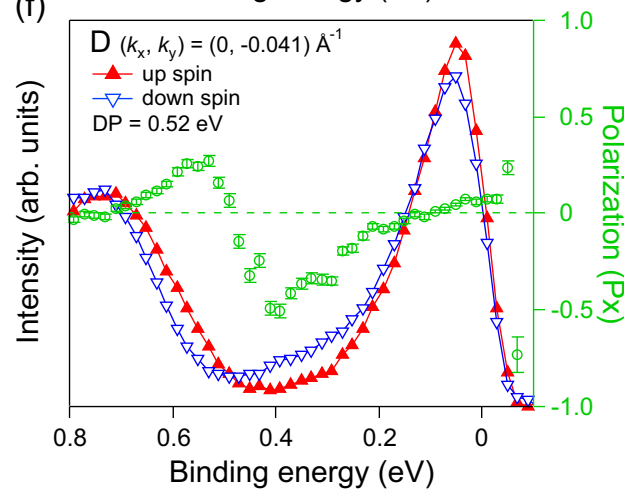

FIG. 8. (a) Schematic image of the ideal helical spin texture of the upper part of the TSS. The measurement points $\left(k_{\mathrm{x}}, k_{\mathrm{y}}\right)$ for SARPES are indicated as A, B, C, and D. (b) ARPES image along the $\bar{\Gamma}-\bar{M}$ direction recorded using a He discharge lamp $(h v=21.2 \mathrm{eV})$. (c)-(f) Spin-resolved energy distribution curves and their spin polarizations at A, B, C, and D as depicted in (a). 
(a)

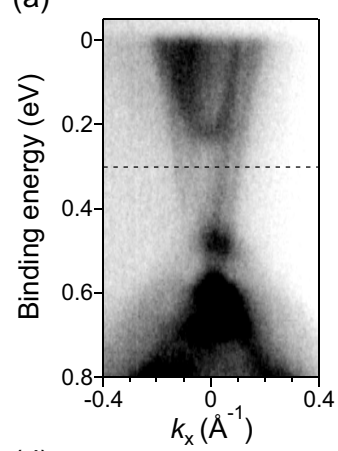

(d)

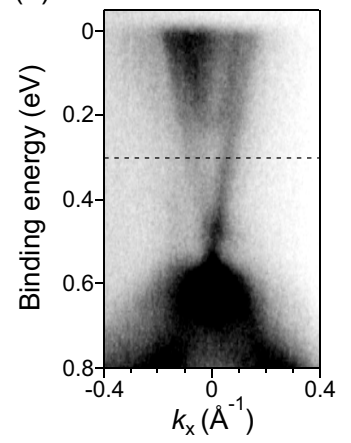

(b)

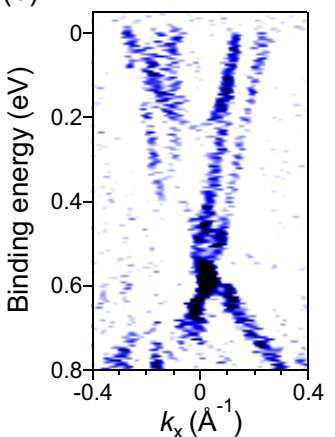

(e)

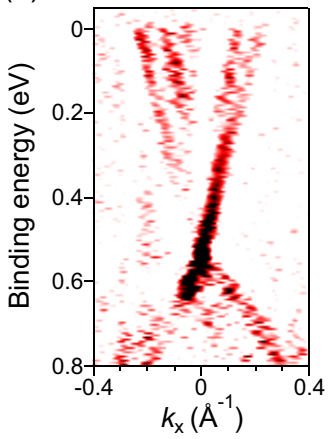

(c)

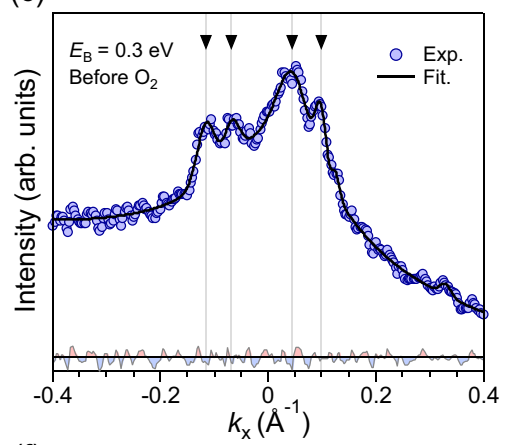

(f)

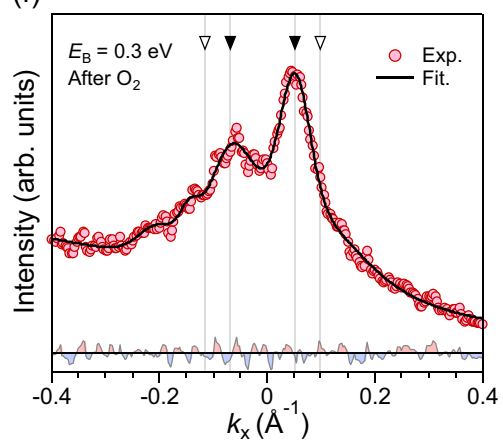

(g)

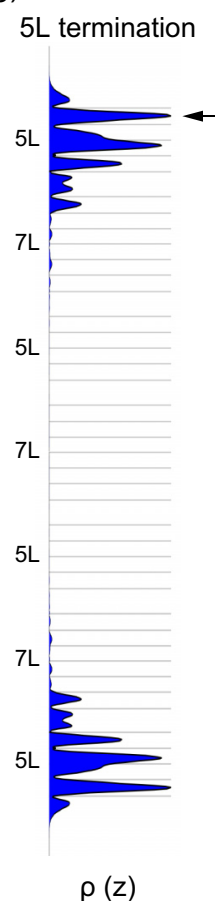

(h)

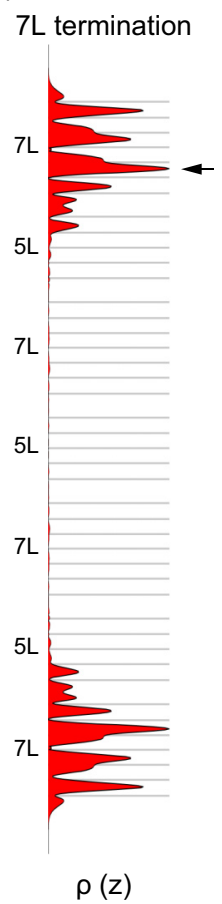

FIG. 9. (a),(b) ARPES image of $\mathrm{PbBi}_{4} \mathrm{Te}_{4} \mathrm{~S}_{3}$ along the $\bar{\Gamma}-\bar{M}$ line recorded at $h v=28 \mathrm{eV}$ and its second derivative. (c) Spin-integrated MDC cut at $E_{\mathrm{B}}=0.30 \mathrm{eV}$ [black dashed line in (a)]. The experimental data is shown with circles and fitted with multiple Gaussian functions. The residuum of the fitting is shown at the bottom of the figure. Inverted triangles indicate the positions of the prominent peaks. Panels (d) and (e) show an ARPES image and its second derivative, respectively, recorded at $h v=28 \mathrm{eV}$ after oxygen exposure. (f) $\mathrm{MDC}$ at $E_{\mathrm{B}}=0.30 \mathrm{eV}$ taken after oxygen exposure. Panels $(\mathrm{g})$ and $(\mathrm{h})$ show the calculated spatial charge densities of the TSSs at $5 \mathrm{~L}$ and $7 \mathrm{~L}$ terminated surfaces, respectively.

[Fig. 6(b)], two Dirac cones are observed within the bulk band gap, similar to that which can be observed with $18 \mathrm{eV}$ [see Fig. 2(b)]. However, at $26 \mathrm{eV}$, the outer Dirac cone shows a higher photoemission intensity than that of the inner Dirac cone [Fig. 6(a)]. Conversely, at $30 \mathrm{eV}$, the inner Dirac cone is enhanced, while the intensity at the outer state is weaker [Fig. 6(c)]. Thus it can be considered that they are independent surface Dirac cones because there is a large difference in excitation energy dependence of the surface state between the outside and the inside. Note that the observed clear dependence on the photon energy appeared in a narrow energy range from 26 to $30 \mathrm{eV}$ and cannot be explained only by the mean free path of the photoelectrons as discussed for $\mathrm{PbBi}_{4} \mathrm{Te}_{7}$ [12]. The observed drastic changes in the photoelectron intensities might be ascribed to the different dipole transition matrix elements for the two surface Dirac cones.

As there has recently been a dispute over the validity of using CDAD spectra to determine the initial state spin textures [30-33,35-37], direct observations of the spin polarizations on the surface states have to be made. This was our motivation to perform the SARPES experiments. Since the photoelectron intensity ratio between the two Dirac cones can be controlled by slightly changing the photon energy, the SARPES measurements were performed using $h v=26$, 28 , and $30 \mathrm{eV}$ with $p$ polarization. Figure 7 shows the experimental geometry, the spin-integrated and spin-resolved MDCs, and their in-plane polarizations $\left(P_{\mathrm{y}}\right)$ taken at $h v \mathrm{~s}=$ 26,28 , and $30 \mathrm{eV}$ for $E_{\mathrm{B}}$ of $0.40,0.30$, and $0.37 \mathrm{eV}$. Here, the spin-up and spin-down spectra are plotted with upright and inverted triangles, respectively. At all photon energies the spin polarization is reversed with respect to the $\bar{\Gamma}$ point. In addition, the sign of the spin polarizations is identical at all photon energies. These results unambiguously show that both Dirac cones can be safely assigned as being independent spin-polarized TSSs.

Moreover, to verify that the observed TSS has a helical spin texture, as schematically shown in Fig. 8(a), the SARPES measurements were performed at several $k$ points. Figure 8(b) shows the ARPES image recorded with a He discharge lamp ( $h v=21.2 \mathrm{eV}$ ). We note that, at $h v=21.2 \mathrm{eV}$, only the inner TSS is observed. Figures 8(c)-8(f) show the spin-resolved energy distribution curves and their spin polarizations. First, we observed position $\mathrm{A}\left(k_{\mathrm{x}}=0.035 \AA^{-1}\right)$ [Fig. 8(c)]. A negative spin polarization is found around $E_{\mathrm{B}}=0.35 \mathrm{eV}$, while a positive spin polarization is found around $E_{\mathrm{B}}=0.55 \mathrm{eV}$. This means that the sign of the spin polarization is reversed across the Dirac point. At position B $\left(k_{\mathrm{x}}=-0.035 \AA^{-1}\right)$ [Fig. 8(d)], the sign of the spin polarization at the TSS is opposite compared with that for position A. These results are in good agreement with the spin-resolved MDCs shown in Figs. 7(c) and $7(\mathrm{~d})$. Similarly, at positions $\mathrm{C}\left(k_{\mathrm{y}}=0.041 \AA^{-1}\right)$ and D $\left(k_{\mathrm{y}}=-0.041 \AA^{-1}\right)$, the signs are also opposite [Figs. 8(e) and 8(f)]. Therefore, the inner TSS has clockwise and counterclockwise helical spin textures at the upper and lower Dirac cones, respectively. The observed spin textures are in good agreement with that obtained by the calculation [Fig. 2(d)]. 
Note that, in the vicinity of $E_{\mathrm{F}}$, we also find a finite spin polarization. In particular, at positions $\mathrm{C}$ and $\mathrm{D}$, the spin polarizations have the opposite sign with respect to the upper part of the Dirac cone, and the absolute values of the spin polarizations $\left(P_{\mathrm{x}}\right)$ are the almost the same. However, at A and $\mathrm{B}$, the sign does not reverse with respect to the $\bar{\Gamma}$ point near the $E_{\mathrm{F}}$. These spin polarizations may originate from the Rashba-like states shown in Fig. 4, but the initial spin polarization is probably modulated by the final state effect $[38,39]$. The final state effect causes $k$-dependent modulations of the spin polarization and strongly depends on the experimental geometry and the polarization of the incident light. In this case, the final state effect largely acts in the $k_{\mathrm{x}}(\bar{\Gamma}-\bar{M})$ direction and causes a negative spin-polarization offset, which masks the information of the initial state.

Finally, to examine whether the TSS is protected by the topmost layer or not, the crystal surface was intentionally exposed to an approximately 100 Langmuir oxygen atmosphere at room temperature. In Figs. 9(a) and 9(b), as well as for panels 9(d) and 9(e), the band dispersions are shown that were taken at $28 \mathrm{eV}$, as well as their second derivatives before and after oxygen exposure, respectively. Figures 9(c) and 9 (f) denote the spin-integrated MDCs at $E_{\mathrm{B}}=0.30 \mathrm{eV}$ shown by the dashed line in Figs. 9(a) and 9(d). Before oxygen exposure, we can clearly see the four prominent peaks derived from two TSSs, represented by inverted triangles [Fig. 9(c)]. However, after oxygen exposure, the outer TSS completely disappeared, while the inner TSS remains visible [Figs. 9(d)-9(f)]. Importantly, the shift of the Dirac point is negligibly small before and after oxygen exposure. This result implies that the outer TSS is localized near the topmost surface, whereas the inner TSS is buried at a deeper site. The disappearance of the outer TSS might not mean that the TSS is killed but indicates that it is relocated into the deeper layers, which results in the absence of the ARPES intensity due to the limited photoelectron mean free path. In this regard, the outer TSS would not be sufficiently protected because adsorbed oxygen molecules are probably unstable. Such an unwanted situation needs to be avoided for applications. To gain more insight into the change caused by oxygen exposure, we calculated the spatial charge densities $\rho(z)$ of the TSSs for the $5 \mathrm{~L}$ and $7 \mathrm{~L}$ terminations at the $\bar{\Gamma}$ point shown in Figs. 9(g) and 9(h). For the 5L terminated surface, the TSS charge density is mostly localized at the topmost $5 \mathrm{~L}$ block, with the largest density between the first and second atomic layers. Conversely, for the 7L terminated surface, the TSS charge density is spread over the $7 \mathrm{~L}$ block, with a substantial penetration into the van der Waals gap below. Note that the center of gravity of the charge density is located slightly deeper than that of the 5L termination, as indicated by the black arrows. The result of this calculation means that the $5 \mathrm{~L}$ terminated surface is more susceptible to the atmosphere than the $7 \mathrm{~L}$ terminated surface, which reasonably explains the experimental result.

\section{CONCLUSION}

In conclusion, the present (S)ARPES study of $\mathrm{PbBi}_{4} \mathrm{Te}_{4} \mathrm{~S}_{3}$ demonstrates the presence of a protected spin-polarized TSS under an oxygen atmosphere. Owing to its wide bulk band gap, high mobility, and robust TSS, $\mathrm{PbBi}_{4} \mathrm{Te}_{4} \mathrm{~S}_{3}$ could thus be suited for application in a spintronic device that would be operable at room temperature.

\section{ACKNOWLEDGMENTS}

The (S)ARPES measurements were performed with the approval of the Proposal Assessing Committee of Hiroshima Synchrotron Radiation Center (Proposals No. 11-A-3, No. 12-A-25, and No. 13-A-30). This work was partly supported by the bilateral collaboration program between RFBR (Russia; No. 15-52-50017) and JSPS (Japan) and also by KAKENHI Grants No. 17H06138 and No. 18H03683. K.S. was financially supported by a Grant-in-Aid for JSPS Fellows (No. 16J03874). The study has also been supported by the Russian Science Foundation (Grant No. 17-12-01047) and RFBR (Grant No. 17-08-00955) for growing the single crystals and characterizing the samples. I.V.S. acknowledges financial support from the Russian Science Foundation (Grant No. 18-12-00169) for band structure calculations and the Ministry of Education and Science of the Russian Federation within governmental program Megagrants (State Task No. 3.9003.2017/9.10) for charge density calculations. This work has been partly performed in the framework of the nanoscience foundry and fine analysis (NFFA-MIUR Italy, Progetti Internazionali) facility. E.V.C. acknowledges financial support from the Saint Petersburg State University Project No. 15.61.202.2015 and the Spanish Ministry of Science and Innovation Grant No. FIS2016-75862-P.
[1] M. Z. Hasan and C. L. Kane, Rev. Mod. Phys. 82, 3045 (2010).

[2] X. L. Qi and S. C. Zhang, Rev. Mod. Phys. 83, 1057 (2011).

[3] Y. Shiomi, K. Nomura, Y. Kajiwara, K. Eto, M. Novak, K. Segawa, Y. Ando, and E. Saitoh, Phys. Rev. Lett. 113, 196601 (2014).

[4] H. Geng, W. Luo, W. Y. Deng, L. Sheng, R. Shen, and D. Y. Xing, Sci. Rep. 7, 3755 (2017).

[5] D. Hsieh, Y. Xia, D. Qian, L. Wray, J. H. Dil, F. Meier, J. Osterwalder, L. Patthey, J. G. Checkelsky, N. P. Ong, A. V. Fedorov, H. Lin, A. Bansil, D. Grauer, Y. S. Hor, R. J. Cava, and M. Z. Hasan, Nature (London) 460, 1101 (2009).
[6] M. Bianchi, D. Guan, S. Bao, J. Mi, B. B. Iversen, P. D. C. King, and Ph. Hofmann, Nat. Commun. 1, 128 (2010).

[7] P. D. C. King, R. C. Hatch, M. Bianchi, R. Ovsyannikov, C. Lupulescu, G. Landolt, B. Slomski, J. H. Dil, D. Guan, J. L. Mi, E. D. L. Rienks, J. Fink, A. Lindblad, S. Svensson, S. Bao, G. Balakrishnan, B. B. Iversen, J. Osterwalder, W. Eberhardt, F. Baumberger, and Ph. Hofmann, Phys. Rev. Lett. 107, 096802 (2011).

[8] H. M. Benia, C. Lin, K. Kern, and C. R. Ast, Phys. Rev. Lett. 107, 177602 (2011). 
[9] M. S. Bahramy, P. D. C. King, A. de la Torre, J. Chang, M. Shi, L. Patthey, G. Balakrishnan, Ph. Hofmann, R. Arita, N. Nagaosa, and F. Baumberger, Nat. Commun. 3, 1159 (2012).

[10] S. V. Eremeev, Yu. M. Koroteev, and E. V. Chulkov, JETP Lett. 92, 161 (2010).

[11] S. V. Eremeev, G. Landolt, T. V. Menshchikova, B. Slomski, Y. M. Koroteev, Z. S. Aliev, M. B. Babanly, J. Henk, A. Ernst, L. Patthey, A. Eich, A. A. Khajetoorians, J. Hagemeister, O. Pietzsch, J. Wiebe, R. Wiesendanger, P. M. Echenique, S. S. Tsirkin, I. R. Amiraslanov, J. H. Dil, and E. V. Chulkov, Nat. Commun. 3, 635 (2012).

[12] T. Okuda, T. Maegawa, M. Ye, K. Shirai, T. Warashina, K. Miyamoto, K. Kuroda, M. Arita, Z. S. Aliev, I. R. Amiraslanov, M. B. Babanly, E. V. Chulkov, S. V. Eremeev, A. Kimura, H. Namatame, and M. Taniguchi, Phys. Rev. Lett. 111, 206803 (2013).

[13] M. Papagno, S. V. Eremeev, J. Fujii, Z. S. Aliev, M. B. Babanly, S. Kr. Mahatha, I. Vobornik, N. T. Mamedov, D. Pacilé, and E. V. Chulkov, ACS nano 10, 3518 (2016).

[14] I. V. Silkin, T. V. Menshchikova, M. M. Otrokov, S. V. Eremeev, Yu. M. Koroteev, M. G. Vergniory, V. M. Kuznetsov, and E. V. Chulkov, JETP Lett. 96, 322 (2012).

[15] T. Okuda, K. Miyamaoto, H. Miyahara, K. Kuroda, A. Kimura, H. Namatame, and M. Taniguchi, Rev. Sci. Instrum. 82, 103302 (2011).

[16] T. Okuda, K. Miyamoto, A. Kimura, H. Namatame, and M. Taniguchi, J. Electron Spectrosc. Relat. Phenom. 201, 23 (2015).

[17] G. Kresse and J. Hafner, Phys. Rev. B 48, 13115 (1993).

[18] G. Kresse and J. Furthmüller, Comput. Mater. Sci. 6, 15 (1993).

[19] J. P. Perdew, K. Burke, and M. Ernzerhof, Phys. Rev. Lett. 77, 3865 (1996).

[20] C. Pauly, G. Bihlmayer, M. Liebmann, M. Grob, A. Georgi, D. Subramaniam, M. R. Scholz, J. Sanchez-Barriga, A. Varykhalov, S. Blügel, O. Rader, and M. Morgenstern, Phys. Rev. B 86, 235106 (2012).

[21] K. Miyamoto, T. Okuda, M. Nurmamat, M. Nakatake, H. Namatame, M. Taniguchi, E. V. Chulkov, K. A. Kokh, O. E. Tereshchenko, and A. Kimura, New J. Phys. 16, 065016 (2014).

[22] See Supplemental Material at http://link.aps.org/supplemental/ 10.1103/PhysRevMaterials.2.104201 for further details of experimental geometry for CD-ARPES measurement and bulk band gap estimation.

[23] H. Liu and L. L. Y. Chang, Am Mineral. 79, 1159 (1994).
[24] D. O. Scanlon, P. D. C. King, R. P. Singh, A. de la Torre, S. McKeown Walker, G. Balakrishnan, F. Baumberger, and C. R. A. Catlow, Adv. Mater. 24, 2154 (2012).

[25] L. L. Wang, M. Huang, S. Thimmaiah, A. Alam, S. L. Bud'ko, A. Kaminski, T. A. Lograsso, P. Canfield, and D. D. Johnson, Phys. Rev. B 87, 125303 (2013).

[26] D. Kong, Y. Chen, J. J. Cha, Q. Zhang, J. G. Analytis, K. Lai, Z. Liu, S. S. Hong, K. J. Koski, S. K. Mo, Z. Hussain, I. R. Fisher, Z. X. Shen, and Y. Cui, Nat. Nanotechnol. 6, 705 (2011).

[27] S. Souma, K. Eto, M. Nomura, K. Nakayama, T. Sato, T. Takahashi, K. Segawa, and Y. Ando, Phys. Rev. Lett. 108, 116801 (2012).

[28] L. Fu, Phys. Rev. Lett. 103, 266801 (2009).

[29] K. Kuroda, H. Miyahara, M. Ye, S. V. Eremeev, Yu. M. Koroteev, E. E. Krasovskii, E. V. Chulkov, S. Hiramoto, C. Moriyoshi, Y. Kuroiwa, K. Miyamoto, T. Okuda, M. Arita, K. Shimada, H. Namatame, M. Taniguchi, Y. Ueda, and A. Kimura, Phys. Rev. Lett. 108, 206803 (2012).

[30] Y. H. Wang, D. Hsieh, D. Pilon, L. Fu, D. R. Gardner, Y. S. Lee, and N. Gedik, Phys. Rev. Lett. 107, 207602 (2011).

[31] W. Jung, Y. Kim, B. Kim, Y. Koh, C. Kim, M. Matsunami, S. I. Kimura, M. Arita, K. Shimada, J. H. Han, J. Kim, B. Cho, and C. Kim, Phys. Rev. B 84, 245435 (2011).

[32] S. R. Park, J. Han, C. Kim, Y. Y. Koh, C. Kim, H. Lee, H. J. Choi, J. H. Han, K. D. Lee, N. J. Hur, M. Arita, K. Shimada, H. Namatame, and M. Taniguchi, Phys. Rev. Lett. 108, 046805 (2012).

[33] H. Mirhosseini and J. Henk, Phys. Rev. Lett. 109, 036803 (2012).

[34] Y. Ishida, H. Kanto, A. Kikkawa, Y. Taguchi, Y. Ito, Y. Ota, K. Okazaki, W. Malaeb, M. Mulazzi, M. Okawa, S. Watanabe, C.T. Chen, M. Kim, C. Bell, Y. Kozuka, H. Y. Hwang, Y. Tokura, and S. Shin, Phys. Rev. Lett. 107, 077601 (2011).

[35] C.-Z. Xu, Y. Liu, R. Yukawa, L.-X. Zhang, I. Matsuda, T. Miller, and T.-C. Chiang, Phys. Rev. Lett. 115, 016801 (2015).

[36] M. R. Scholz, J. Sanchez-Barriga, J. Braun, D. Marchenko, A Varykhalov, M. Lindroos, Y. J. Wang, H. Lin, A. Bansil, J. Minár, H. Ebert, A. Volykhov, L. V. Yashina, and O. Rader, Phys. Rev. Lett. 110, 216801 (2013).

[37] J. Sanchez-Barriga, A. Varykhalov, J. Braun, S.-Y. Xu, N. Alidoust, O. Kornilov, J. Minár, K. Hummer, G. Springholz, G. Bauer, R. Schumann, L. V. Yashina, H. Ebert, M. Z. Hasan, and O. Rader, Phys. Rev. X 4, 011046 (2014).

[38] C.-H. Park and S. G. Louie, Phys. Rev. Lett. 109, 097601 (2012).

[39] H. Wortelen, H. Mirhosseini, K. Miyamoto, A. B. Schmidt, J. Henk, and M. Donath, Phys. Rev. B 91, 115420 (2015). 